\title{
Correction to: An Initial Experience of Shoulder Resections and Reconstruction for Bone Tumours from a Cancer Centre in North-East India
}

\author{
Gaurav Das ${ }^{1}$. Srinivas Bannoth ${ }^{1}$ - Bibhuti Bhusan Borthakur ${ }^{1} \cdot$ Sumanjit Boro ${ }^{2} \cdot$ Revanth Kumar $^{1} \cdot$ Pritesh Singh $^{1}$. \\ Jitin Yadav ${ }^{1}$
}

Published online: 19 May 2021

(C) Indian Association of Surgical Oncology 2021

\section{Correction to: Indian Journal of Surgical Oncology}

$$
\text { https://doi.org/10.1007/s13193-021-01292-x }
$$

There is misrepresentation of details of the last patient in table1 (i.e 10 year male with Ewings sarcoma who has undergone vascularised fibular graft). Patient had expired on 9 th postoperative month (status- dead). This patient developed both local recurrence and distant metastasis (LR: Yes, DM: Yes).

Publisher's Note Springer Nature remains neutral with regard to jurisdictional claims in published maps and institutional affiliations.

The online version of the original article can be found at https:/doi.org/ 10.1007/s13193-021-01292-x

Srinivas Bannoth

srinivasbannoth@gmail.com

Gaurav Das

drdas.gaurav@gmail.com

Bibhuti Bhusan Borthakur

Borthakur.bb@gmail.com

Sumanjit Boro

Sumanjit.boro@yahoo.in

Revanth Kumar

drrevanth@gmail.com
Pritesh Singh

Singhpritesh17@gmail.com

Jitin Yadav

jitindrcool@gmail.com

1 Department of Surgical Oncology, DR.B. Borooah Cancer Institute, A.K. Azad Road, Gopinath Nagar, Guwahati, Assam, India

2 Department of Plastic surgery, DR.B. Borooah Cancer Institute, Guwahati, India 